\title{
Some highlights of the latest CoRoT results on stellar physics
}

\author{
S. Deheuvels ${ }^{1,2}$ and the $\operatorname{CoRoT}$ team \\ ${ }^{1}$ Université de Toulouse; UPS-OMP; IRAP; Toulouse, France \\ email: sebastien.deheuvels@irap.omp.eu \\ ${ }^{2}$ CNRS; IRAP; 14, avenue Edouard Belin, F-31400 Toulouse, France
}

\begin{abstract}
Since its launch in December 2006, the CoRoT satellite has provided photometric data precise down to the micro-magnitude level for about 150 bright stars and 150000 fainter ones. These stars have been observed over runs covering up to 160 days with a $90 \%$ duty cycle. Seismic data of such precision had been longed for by the scientific community for decades, and expected as a way of making progress in our understanding of stellar structure and evolution. The analysis and interpretation of CoRoT seismic data have indeed made it possible to place observational constraints on several key aspects of stellar structure and evolution, such as the size of mixed convective cores, magnetic activity, mass loss... We here present some highlights of the CoRoT results and their implications in terms of internal stellar structure.
\end{abstract}

\section{The CoRoT mission}

After almost six years of observations, the CoRoT satellite has observed 156 bright stars $\left(5.4 \leqslant m_{V} \leqslant 10.5\right)$, and more than 160000 fainter ones $\left(11 \leqslant m_{V} \leqslant 16\right)$ that lie at the intersection of the Galactic plane and the equator. In November 2012, the satellite encountered an electric breakdown, which could unfortunately not be repaired despite great efforts. CoRoT yielded seismic data of unprecedentedly high quality by observing over long observation runs (ranging from about 20 days to more than 160 days) with duty cycles over $90 \%$. This led to several breakthroughs such as the precise characterization of solar-like oscillation modes in stars other than the Sun (Appourchaux et al. 2008, Michel et al. 2008), the detection of mixed modes in several thousands of red giants (De Ridder et al. 2009, Mosser et al. 2011), the evidence for a correlation between oscillations and the occurrence of an outburst in a Be star (Huat et al. 2009), the detection of solar-like oscillations in massive stars (Belkacem et al. 2010, Degroote et al. 2010), the detection of a magnetic activity cycle in the solar-like pulsator HD 49933 (García et al. 2010)... Several reviews already presented these results from CoRoT, along with many others (Michel \& Baglin 2012, Baglin et al., in preparation). We here give a description of some of the latest results that were obtained with CoRoT data, focusing on the main scientific objectives of the mission: making progress in our understanding of stellar convection and of the effects of rotation on the structure and evolution of stars.

\section{Rotation}

Reaching a better understanding of the effects of rotation on stellar structure and evolution was one of the main objectives of the CoRoT mission. Progress in this field has been hindered by the lack of observational constraints on the rotation profiles of stars. By providing quasi-uninterrupted photometric observations over periods of several months, the CoRoT satellite offered the opportunity to measure rotation periods both 


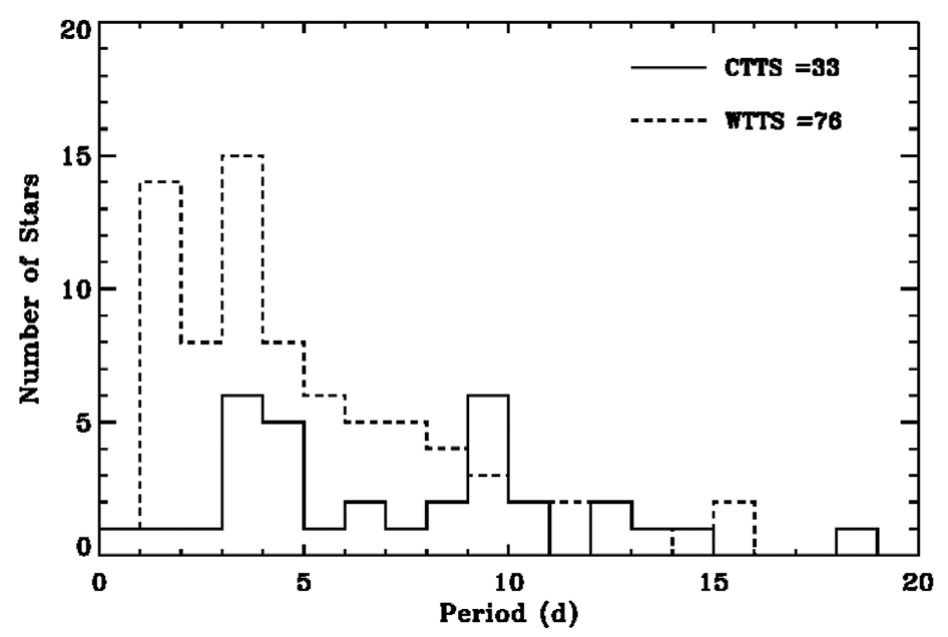

Figure 1. Distribution of rotation periods of weak-line T-Tauri stars and classical T-Tauri stars in the NGC2264 star forming region (figure from Affer et al. 2013).

from stellar activity and from seismology for stars with different masses, from the premain sequence to more advanced stages. Also, one of the objectives of $\operatorname{CoRoT}$ was to help us understand the oscillation spectra of fast rotators whose great complexity have severely limited our seismic diagnostics for these stars so far.

\subsection{Rotation periods of T-Tauri stars in NGC2264}

The star forming region NGC 2264, which is one of the best known in the solar vicinity, falls in the field of view of CoRoT. This gave a unique opportunity to study young stars still in a formation phase, and in particular to estimate their rotation periods. This is particularly relevant to study the role of disk locking (magnetic interaction between young stars and their accretion disk) in the braking of stars during the pre-main sequence contraction and thus to better understand the evolution of angular momentum at this time. If disk locking is indeed responsible for the existence of a slow-rotating population in young clusters, there should be a correlation between accretion and stellar rotation. Several previous studies had failed to detect such correlation (e.g. Stassun et al. 1999, Cieza \& Baliber 2006). Using CoRoT observations of the cluster NGC 2264, Affer et al. (2013) have measured the rotation periods of both classical T-Tauri stars (CTTS), which are assumed to have an accretion disk, and weak T-Tauri stars (WTTS), which have no disk. They found that the two populations have significantly different rotation distributions with the WTTS rotating faster than the CTTS (Fig. 1). This suggests that the presence of accretion affects the rotational period and is consistent with the disk-locking scenario.

\subsection{Surface rotations of low-mass main-sequence stars}

By observing stars almost continuously over periods of several months, CoRoT made it possible to reach the level of precision and the frequency resolution required to measure rotational splittings in the oscillation spectra of solar-like stars. For instance, a mean rotational splitting for $\mathrm{p}$ modes has been estimated in the F star HD 181420 (Barban et al. 2009). This measure was found consistent with the surface rotation period inferred from the periodic modulation of the lightcurve attributed to starspots.

Another particularly interesting CoRoT target is the K-type solar-like pulsator HD 52265. This star was selected as a primary target because it hosts a planetary companion, 


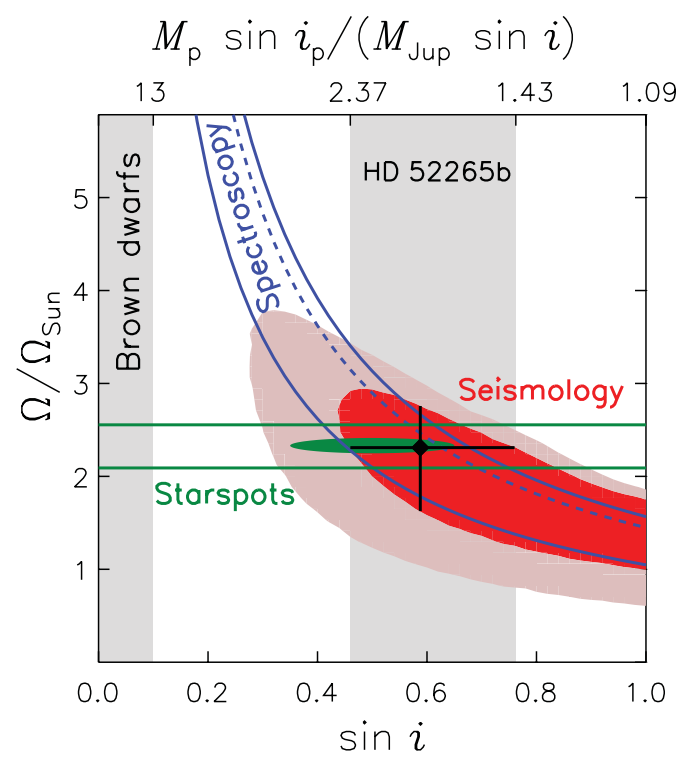

Figure 2. Constraints on the surface rotation of HD 52265 from seismology (black diamond with 1- $\sigma$ error bars), from spectroscopy (blue lines) and from the modulation of the lightcurve caused by star spots (horizontal green lines). Figure taken from Gizon et al. (2013), see this reference for more information.

and it was observed during 117 days by CoRoT. A modeling of the star was performed by Escobar et al. (2012) and yielded precise estimates of its mass and age. Recently, Gizon et al. (2013) obtained an unambiguous measurement of the average surface rotation of the star by confronting three different measurements:

- The signature of starspot modulation, which was found in the CoRoT lightcurve of the star (Ballot et al. 2011).

- The mean rotational splitting of the acoustic modes, which was extracted from the oscillation spectrum of the star by Gizon et al. (2013).

- The combination of the spectroscopic $v \sin i$, the radius of the star from the seismic modeling, and its inclination angle $i$, which could be determined from seismology (Gizon et al. 2013).

The results of these three methods are remarkably consistent, as shown by Fig. 2 .

It is well known that low-mass stars are braked during the main sequence because they lose angular momentum through a magnetized wind generated by the convective envelope. Having access to precise and reliable rotation periods for stars whose mass and age can also be constrained by seismology, as was the case for HD 52265, could give precious observational constraints to calibrate theoretical laws of angular momentum loss (e.g. Kawaler 1988), which could be helpful for gyrochronology.

\subsection{Fast rotation in intermediate-mass main-sequence stars}

Intermediate-mass stars that fall in the $\delta$ Scuti Instability Strip have extremely rich oscillation spectra. Hundreds of modes were detected in the oscillation spectra of several CoRoT $\delta$ Scuti stars (e.g. Poretti et al. 2009, García Hernández et al. 2009). Moya \& Rodríguez-López (2010) showed that $\delta$ Scuti stars have in principle enough energy to excite such a large number of modes.

The complexity of the observed spectra of $\delta$ Scuti stars is probably related to their fast rotational velocities. Indeed, these stars are not braked by a magnetic wind during the 
main sequence and they can rotate at non-negligible fractions of the break-up velocity. Both theoretical and numerical works have been led to explore the impact of such fast rotation on the mode frequencies, involving the integration of the oscillation equations taking rotation into account in 2D models (Lignières et al. 2006, Reese et al. 2006). These studies showed that for fast rotators, two frequency subsets should be visible: a regular one, which can be approximated by an asymptotic theory analogous to the slowrotation case (Pasek et al. 2012), and an irregular one, with specific statistic properties. This was proposed as an explanation for the extreme richness of the spectra of $\delta$ Scuti stars. Despite the large number of expected modes, recent theoretical work predicts that equidistances corresponding to the large separation and to twice the rotational splitting should still be identifiable in the frequency distribution (Lignières et al. 2010), giving hope to interpret the spectra of these stars.

García Hernández et al. (2013) analyzed the CoRoT lightcurve of the $\delta$ Scuti star HD 174966 and extracted 185 significant independent frequencies. They found a significant periodicity of about $64 \mu \mathrm{Hz}$ in the frequency set and built an échelle diagram of the detected frequencies folded with this equidistance. By using spectroscopic measurements, they derived an upper limit of $29 \mu \mathrm{Hz}$ for the rotational splitting and concluded that the observed periodicity is more likely to correspond to the large separation of the star. Paparó et al. (2013) also found regular spacings in the mode pattern of the $\delta$ Scuti star CoRoT 102749568, but it remains uncertain whether they are caused by rotational effects or to the large separation of $\mathrm{p}$ modes. These studies constitute the first step toward the understanding of the complex spectra of fast rotators and give the perspective of measuring the mean densities of $\delta$ Scuti stars regardless of their rotational velocities.

\subsection{Rotation periods of CoRoT subgiants}

Estimating the surface rotation of post-main sequence stars can place valuable constraints on the mechanisms of angular momentum transport inside stars, which remain poorly understood. Indeed, the rotation periods of subgiants and red giants are the result of a competition between the expansion of the star which tends to spin it down and the deepening of the convective envelope, which dredges up material from the core and tends to spin up the envelope. Redistribution of angular momentum inside the star also influences the surface rotation.

By searching for periodicity in the lightcurves of CoRoT subgiants, do Nascimento et al. (2013) obtained rotation periods ranging from 30 to 100 days for 30 of these targets. They also found that these observed periods are consistent with the surface rotations predicted by stellar evolution models that include rotationally-induced transport of angular momentum as described by Zahn (1992). However, recent observations from the Kepler satellite yielded estimates of the core rotation of subgiants (Deheuvels et al. 2012) and red giants (Beck et al. 2012, Mosser et al. 2012), which showed that the transport of angular momentum à la Zahn (1992) is not efficient enough and that another mechanism must be at work (e.g. Marques et al. 2013). The origin of this extra mechanism is not yet known, but a more efficient internal transport of angular momentum should imply faster envelope rotation. On the other hand, van Saders \& Pinsonneault (2013) recently showed that the rotation periods found by do Nascimento et al. (2013) are smaller than would be expected if solid-body rotation at all times (i.e. instantaneous transport of angular momentum) is assumed, which is consistent with the detection of differential rotation in these stars. 


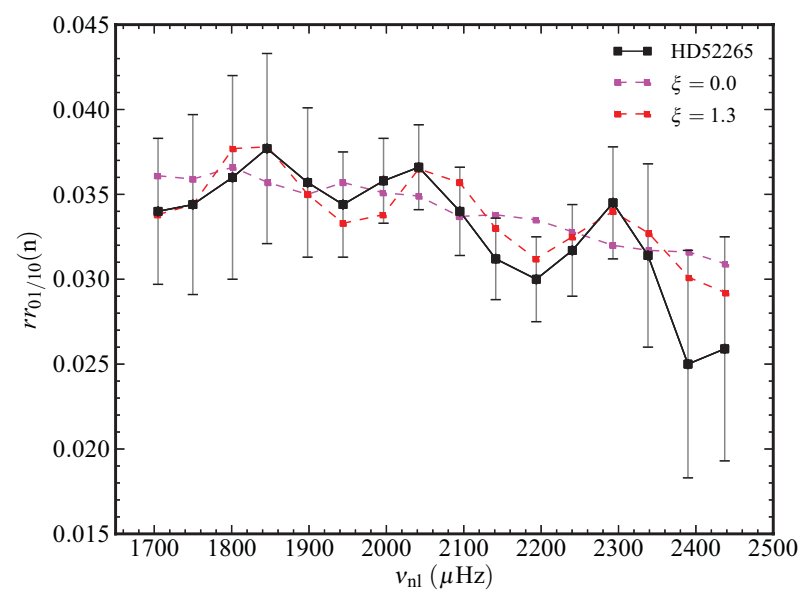

Figure 3. Variations in the frequency ratios $r r_{01}$ and $r r_{10}$ (see Roxburgh \& Vorontsov 2003 for a definition) of the solar-like pulsator HD 52265 (black circles). The colored lines indicate the frequency ratios of the best-fit models (purple: no overshooting, red: overshooting over a distance of $0.95 H_{p}$ ). (figure from Lebreton \& Goupil 2012)

\section{Convection}

\subsection{Core and envelope overshooting}

Several processes are expected to extend the size of convective regions beyond the Schwarzschild frontier (overshooting, rotational mixing, ...), but since we lack a realistic description of these mechanisms, the actual size of convective zones remains uncertain. This generates large uncertainties in our determination of stellar ages for stars that have a convective core. Observational constraints on the size of convective regions are therefore needed.

Core overshoot. CoRoT observations have produced indications in favor of an extension of the mixed core for stars of different masses and evolutionary stages, and using different seismic diagnostics. Since these results have already been presented by Michel \& Baglin (2012), we only briefly recall them here. For the main-sequence solar-like pulsator HD 49933, Goupil et al. (2011) found that an extension of the mixed core over a distance of about $0.2 H_{p}$ is required to reproduce the observed frequency separation $\delta \nu_{01}$. The authors showed that rotational mixing as it is currently modeled cannot account in itself for such a large extension, suggesting that core overshoot is needed. On the other hand, Escobar et al. (2012) found that no extra mixing beyond the convective core is required for the main-sequence star HD 52265. Since this star has a higher abundance of heavy elements than HD 49933, this result raises the question of a possible dependency of core overshooting on metallicity. By using mixed modes, which are sensitive to the chemical composition of the core, Deheuvels \& Michel (2011) found that models with a core overshoot between 0.18 and $0.2 H_{p}$ best reproduce the observed frequencies, but they could not exclude the case of a very small overshoot below $0.05 H_{p}$. Evidence for extended convective cores was also obtained for more massive stars. Degroote et al. (2010) detected a deviation from the regular spacing in period of gravity modes in the B star HD 50230, which they could explain only by assuming a core overshoot above $0.2 H_{p}$. Neiner et al. (2012) detected groupings of modes in two Be stars at the end of the main sequence and they showed that a core extended over at least $0.3 H_{p}$ is needed to explain both the excitation of the modes and the frequencies of the observed mode groupings. 

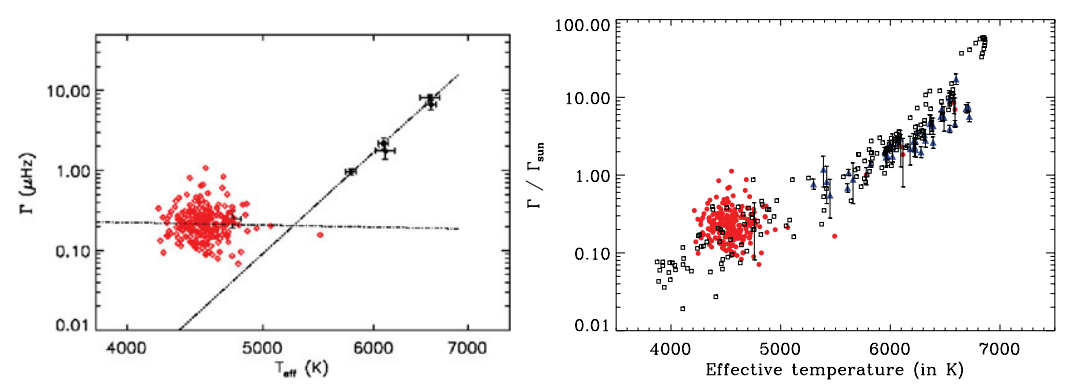

Figure 4. Left: Measured mode linewidth versus $T_{\text {eff }}$ for CoRoT main-sequence (black diamonds) and red-giant (red diamonds) solar-like pulsators (from Baudin et al. 2011). Right: Mode linewidths predicted from non-adiabatic calculations (open squares) versus $T_{\text {eff }}$. The blue triangles represent main-sequence stars observed with Kepler, and red circles correspond to red giants observed with CoRoT(from Belkacem et al. 2012).

Envelope overshoot. Overshooting from the convective envelope has a less direct impact on stellar evolution than core overshooting, but it can help us understand this theoretically challenging phenomenon. In favorable cases, the depth of the boundary of the convective envelope can be estimated through seismology because the abrupt transition between convective and radiative energy transport induces a glitch in the sound speed profile, to which acoustic modes are sensitive. Lebreton \& Goupil (2012) showed that the observed seismic ratios $r r_{01}$ and $r r_{10}$ (as introduced by Roxburgh \& Vorontsov 2003) of the CoRoT solar-like pulsator HD 52265 are reproduced at closest when assuming an envelope overshooting extending over $0.95 H_{p}$ (see Fig. 3). By comparison, ChristensenDalsgaard et al. (2011) recently showed that an overshooting over a distance of $0.37 \mathrm{H}_{p}$ is needed at the base of the convective envelope of the Sun. The difference between the overshooting distances of the two stars might be linked to the fact that HD 52265 has roughly twice the abundance of heavy elements of the Sun.

\subsection{Convection in the super-adiabatic layer}

The structure of the outer convective envelope remains poorly understood because convective transport is inefficient in this region, which makes the mixing length theory inappropriate. This is currently a major problem for seismology because the frequencies of individual modes depend on the structure of the super-adiabatic layer. Empirical corrections of these so-called near surface effects have been proposed (Kjeldsen et al. 2008) but a better understanding of the super-adiabatic layer is needed to solve this problem. The seismic data from CoRoT and now Kepler made it possible to measure the amplitudes and linewidths of solar-like pulsators other than the Sun. This can yield valuable observational constraints on the super-adiabatic layer because the mode amplitudes and widths depend on the excitation and damping of the modes.

Mode amplitudes. Several scaling laws have been proposed for the mode amplitudes of main-sequence solar-like pulsators (e.g. Houdek et al. 1999, Samadi et al. 2007). They predict mode amplitudes to scale with $(L / M)^{s}$, where $s$ ranges from 0.7 to 1.5. CoRoT observations are in agreement with this scaling and favor values of $s$ in the lower end of the interval [0.7, 1.5] (Baudin et al. 2011). Detailed comparisons between 3D simulations and CoRoT observations were performed for the solar-like pulsator HD 49933 by Samadi et al. (2010). They showed that the effects of metallicity must be taken into account, and while they could reproduce the observed amplitudes for low-frequency modes, differences remain at higher frequency. 
The detection of oscillations in thousands of red giants with CoRoT and Kepler gave the opportunity to test whether or not these scaling relations can be extended to the red-giant branch. Baudin et al. (2011) extracted mode amplitudes for several hundreds of CoRoT red giants. To compare the mode amplitudes that are predicted from 3D simulations with CoRoT observations, a velocity-intensity relation is needed. Samadi et al. (2012) showed that for red-giant stars, non-adiabatic effects need to be taken into account in this conversion. Even then, the predicted mode amplitudes for red giants are underestimated by about $40 \%$. Solving this discrepancy will probably require a better knowledge of the depth at which the mode inertia need to be computed and a more realistic treatment of the interaction between convection and pulsations (Samadi et al. 2012).

Mode linewidths. Baudin et al. (2011) also extracted the mode linewidths for the solar-like pulsators observed by CoRoT. They found that the mode linewidths of mainsequence pulsators vary very sharply with the star's temperature $\left(\Gamma \propto T_{\text {eff }}^{m}\right.$ with $m=$ $16.2 \pm 2$ ), which was later confirmed by Appourchaux et al. (2012) with Kepler data. This sheds new light on the unexpectedly large width of the modes of $\mathrm{F}$ stars such as HD 49933 (Appourchaux et al. 2008). According to Baudin et al. (2011), this scaling law does not extend to red giants (see Fig. 4, left panel), which led them to suggest that a different damping mechanism might be at work in these stars. However, Belkacem et al. (2012) recently performed fully non-adiabatic calculations with the MAD code (Dupret 2001) including time-dependent convection, and they could reproduce the observed mode linewidths of both Kepler main-sequence stars and CoRoT red giants (see Fig. 4, right panel). Kepler observations, which are longer than those of CoRoT, should yield mode linewidth for red giants higher in the red-giant branch $\left(T_{\text {eff }}\right.$ lower than $\left.4200 \mathrm{~K}\right)$, which will make it possible to test the predictions of Belkacem et al. (2012).

\section{Conclusion}

The CoRoT data have now brought contributions in many different fields of stellar physics. CoRoT is providing us with valuable observational constraints on several physical processes that are theoretically challenging, such as the evolution of rotation profiles with time, the transport of angular momentum in stars, core and envelope overshooting, the properties of convection in the super-adiabatic layer... CoRoT leaves the scientific community with an extremely rich seismic data set, which has certainly not been used to its full potential yet. The reason for this is of course the very large number of targets that were observed, but also the difficulties that we currently encounter in identifying the observed modes in many cases, or in interpreting the mode frequencies due to nearsurface effects. It is exciting to see that we are now making progress in these domains, with for instance spectroscopic ground-based follow-up campaigns that can provide mode identification, interesting developments in our understanding of the oscillation spectra of fast rotators, or the prospect to better describe the structure of the super-adiabatic layers using the constraints given by the amplitudes and lifetimes of solar-like modes. This shows that new scientific breakthroughs can definitely be expected from CoRoT data in the years to come.

\section{Acknowledgements}

I am very grateful to E. Michel and A. Baglin for their advices and help to write this review. 


\section{References}

Affer, L., Micela, G., Favata, F., Flaccomio, E., \& Bouvier, J. 2013, MNRAS, 430, 1433

Appourchaux, T., Michel, E., Auvergne, M., et al. 2008, A\&BA, 488, 705

Appourchaux, T., Benomar, O., Gruberbauer, M., et al. 2012, A\&GA, 537, A134

Ballot, J., Gizon, L., Samadi, R., et al. 2011, A\&A, 530, A97

Barban, C., Deheuvels, S., Baudin, F., et al. 2009, A\&A, 506, 51

Baudin, F., Barban, C., Belkacem, K., et al. 2011, A\&\&A, 535, C1

Beck, P. G., Montalbán, J., Kallinger, T., et al. 2012, Nature, 481, 55

Belkacem, K., Dupret, M. A., \& Noels, A. 2010, A\& A, 510, A6

Belkacem, K., Dupret, M. A., Baudin, F., et al. 2012, A\& A, 540, L7

Christensen-Dalsgaard, J., Monteiro, M. J. P. F. G., Rempel, M., \& Thompson, M. J. 2011, MNRAS, 414, 1158

Cieza, L. \& Baliber, N. 2006, ApJ, 649, 862

De Ridder, J., Barban, C., Baudin, F., et al. 2009, Nature, 459, 398

Degroote, P., Aerts, C., Baglin, A., et al. 2010, Nature, 464, 259

Deheuvels, S. \& Michel, E. 2011, A\& A, 535, A91

Deheuvels, S., García, R. A., Chaplin, W. J., et al. 2012, ApJ, 756, 19

do Nascimento, Jr., J.-D., Takeda, Y., Meléndez, J., et al. 2013, ApJ, 771, L31

Dupret, M. A. 2001, $A \mathscr{E} A, 366,166$

Escobar, M. E., Théado, S., Vauclair, S., et al. 2012, A\&A, 543, A96

García, R. A., Mathur, S., Salabert, D., et al. 2010, Science, 329, 1032

García Hernández, A., Moya, A., Michel, E., et al. 2009, A\&\&A, 506, 79

García Hernández, A., Moya, A., Michel, E., et al. 2013, A\&̛A, 559, A63

Gizon, L., Ballot, J., Michel, E., et al. 2013, Proc. of the Nat. Acad. of Sciences, 110, 13267

Goupil, M. J., Lebreton, Y., Marques, J. P., et al. 2011, Journal of Physics: Conference Series, 271,012032

Houdek, G., Balmforth, N. J., Christensen-Dalsgaard, J., \& Gough, D. O. 1999, A\&A, 351, 582

Huat, A.-L., Hubert, A.-M., Baudin, F., et al. 2009, A\& A, 506, 95

Kawaler, S. D. 1988, ApJ, 333, 236

Kjeldsen, H., Bedding, T. R., \& Christensen-Dalsgaard, J. 2008, ApJ, 683, L175

Lebreton, Y. \& Goupil, M. J. 2012, A\& A, 544, L13

Lignières, F., Rieutord, M., \& Reese, D. 2006, $A \& A$, 455, 607

Lignières, F., Georgeot, B., \& Ballot, J. 2010, AN, 331, 1053

Marques, J. P., Goupil, M. J., Lebreton, Y., et al. 2013, A\&A, 549, A74

Michel, E. \& Baglin, A. 2012, arXiv: 1202.1422

Michel, E., Baglin, A., Auvergne, M., et al. 2008, Science, 322, 558

Mosser, B., Barban, C., Montalbán, J., et al. 2011, A\&A, 532, A86

Mosser, B., Goupil, M. J., Belkacem, K., et al. 2012, A\& A, 548, A10

Moya, A. \& Rodríguez-López, C. 2010, ApJ, 710, L7

Neiner, C., Mathis, S., Saio, H., et al. 2012, A\& A, 539, A90

Paparó, M., Bognár, Z., Benkő, J. M., et al. 2013, A\&̈A, 557, A27

Pasek, M., Lignières, F., Georgeot, B., \& Reese, D. R. 2012, A\&4A, 546, A11

Poretti, E., Michel, E., Garrido, R., et al. 2009, $A \& A$, 506, 85

Reese, D., Lignières, F., \& Rieutord, M. 2006, A\&A, 455, 621

Roxburgh, I. W. \& Vorontsov, S. V. 2003, A\&A, 411, 215

Samadi, R., Georgobiani, D., Trampedach, R., et al. 2007, A\&SA, 463, 297

Samadi, R., Ludwig, H.-G., Belkacem, K., Goupil, M. J., \& Dupret, M.-A. 2010, A\& A, 509, A15

Samadi, R., Belkacem, K., Dupret, M.-A., et al. 2012, A\&A, 543, A120

Stassun, K. G., Mathieu, R. D., Mazeh, T., \& Vrba, F. J. 1999, AJ, 117, 2941

van Saders, J. L. \& Pinsonneault, M. H. 2013, ApJ, 776, 67

Zahn, J.-P. 1992, A\&A, 265, 115 